\title{
O conceito de espaço na epidemiologia das doenças infecciosas
}

\author{
The concept of space in infectious disease \\ epidemiology
}

Luiz Jacintho da Silva 1

\footnotetext{
1 Departamento de Clínica Médica. Faculdade de Ciências Médicas, Universidade Estadual de Campinas.

C. P. 6.019 , Campinas, SP 13081-970, Brasil.

luisjs@correi onet.com.br
}

\begin{abstract}
A bstract This articleanalyzes how space is employed in infectious disease epidemiology, with a brief retrospective of the various definitions of space and its implications. Emphasis is given to the theory of natural foci of infectious di seases formulated by Pavlovsky and the interactions of epidemi ol ogy and geography. The current problem posed by emerging infections is seen as a determinant of the need for further discussion on the concept of space in infectious disease epidemiology.

Key words Epidemiology; Geography; Ecology; Infectious Disease
\end{abstract}

Resumo Análise teórica da utilização do concei to de espaço na epidemiologia das doenças infecci osas. Faz-se uma breve retrospectiva do uso de diferentes conceituações de espaço e de suas implicações. Ênfase é dada à teoria dos focos naturais de Pavlovsky e às interações entre a epidemiologi a ea geografia. O problema atual das infecções emergentes é visto como determinante da necessi dade de se intensificarem as discussões sobre este concei to na epidemi ologia das doenças infecciosas.

Palavras-chave Epidemiologia; Geografia; Ecologia; Doenças Infecciosas 


\section{Introdução}

A utilização do espaço como categoria de análise para a compreensão da ocorrência e distribuição das doenças nas coletividades é anterior ao surgimento da epidemiologia como disciplina científica. As primeiras análises já incorporavam o conceito de espaço. A percepção de que determinadas doenças ocorriam preferencialmente neste ou naquele lugar é antiga. De Hipócrates (Buck et al., 1988) aos primeiros epidemiologistas (Snow, 1990), o diferencial de doenças conforme o local vem sendo objeto de interesse. Apesar disso, os conceitos utilizados eram implícitos, nunca discutidos per si (Verhasselt, 1981; Wilding et al., 1995).

A epidemiologia descritiva, conforme a conceituação clássica, entende o espaço como um conjunto de determinantes, geralmente de natureza biológica ou natural, como clima, vegetação, latitude (Heunis et al., 1995; Sinha \& Benedict, 1996) e topografia (Forattini, 1976). Não há dúvidas de que o clima sempre foi o determinante a receber maior atenção, tendo seu interesse renovado graças ao progressivo aquecimento gl obal (Gill, 1920a e b; Peixoto, 1975; Lovejoy, 1993; Burgos et al., 1994). Em época mais recente, a poluição ambiental e outros fatores físicos, como quantidade de radiação ultravioleta ou intensidade de campo eletromagnético, vêm atraindo $a$ atenção, principalmente à medida que os epidemiologistas se voltam cada vez mais para as neoplasias.

\section{A teoria dos focos naturais e a vertente ecológico-geográfica}

Possivelmente a primeira apreciação teórica do conceito de espaço aplicado à epidemiologia foi feita por Pavlovsky, parasitologista russo, que, na década de 1930, desenvolveu a teoria dos focos naturais das doenças transmissíveis, também conhecida como teoria da nidalidade natural das doenças transmissíveis (Pavlovsky, s.d., a e b). $\mathrm{O}$ grande incentivo ao desenvolvimento de sua teoria foi o avanço da fronteira agrícola soviética no início da era Stalin. Extensas áreas do território da então União Soviética, particularmente na Ásia, estavam sendo desbravadas e exploradas, tanto para a agricultura, quanto por seus recursos naturais, como madeira e minerais. Como conseqüência, surgiram alguns problemas de saúde pública, como a leishmaniose na Ásia Central e as encefalites por arbovírus na Sibéria - interessante notar que passados cinqüenta anos, a preocupação da epidemiologia com as novas fronteiras continua presente (Coimbra et al., 1984; Morse, 1995). Uma pesquisa na base de dados $\mathrm{Me}$ dline, utilizando o termo natural foci de 1985 a 1996, trará um grande número de artigos, mas a totalidade foi publicada na Rússia ou em países que em algum momento estiveram sob influência soviética, como a China e as antigas repúblicas da Tchecoslováquia e lugoslávia.

Pavlovsky desenvolveu uma teoria de marcado cunho ecologista, mas cujo grande mérito foi o de estabelecer o conceito de que o espaço era o cenário no qual circulava o agente infeccioso - a patobiocenose; este cenário era classificado em natural, ou intocado pela ação humana, e antropopúrgico, alterado pela ação humana. Pavlovsky inegavelmente estava preocupado em desenvolver uma metodologia de cunho eminentemente prático, como se pode depreender de um trecho de seu livro, no capítulo 7:

"Medical aspects of landscape study, The study of landscapes is of great importance for medicinein view of its specific aim, in particular, to find out the presence of natural foci of diseases in a given landscape area, characterized by its general geobotanical aspect; this is the primary aim. The second, no less important, aim is to ascertain man's influence in the pre sent epoch or in the historical past on the primordial state of a particular landscape" (Pavlovsky, s.d., a).

A modificação do espaço, ou paisagem, determinava alterações ecológicas na patobiocenose, alterando a circulação do agente infeccioso. Ainda que a teoria dos focos naturais de Pavlovsky seja por demais restrita para as necessidades atuais da epidemiologia, o modelo do foco natural e da sua transformação pela ação humana com conseqüente alteração da epidemiologia de uma doença é fundamental para a análise do espaço enquanto categoria da epidemiologia. O espaço, na sua conceituação clássica em epidemiologia, é apenas o substrato que exerce sua influência através de fenômenos naturais, como o clima. Segundo essa conceituação, o espaço é estático, imutável, um espectador não participante (Sabroza et al., 1996).

A teoria dos focos naturais recebeu uma atenção limitada, principalmente devido à II Guerra Mundial, que sobreveio logo após, dificultando a difusão das idéias. Mesmo assim, muitos parasitologistas adotaram a teoria de Pavlovsky, mas não a expandiram, utilizaramna apenas dentro do seu val or aparente. Cabe destacar os trabalhos de Audy (Audy, 1958), parasitologista britânico, preocupado com a Rickettsia tsutsugamuchi, no sudeste asiático, e os 
trabalhos de parasitologistas brasileiros, principalmente da escola de Samuel Pessoa (Barreto, 1967; Pessoa, 1978).

A crescente urbanização verificada em todo o mundo e particularmente no terceiro mundo diminuiu o interesse pela teoria dos focos naturais, uma vez que a natureza intocada pela ação humana tornou-se praticamente inexistente, e a teoria de Pavlovsky, tal como foi enunciada, dá conta apenas das primeiras fases da transformação dos focos naturais. Verdade que surtos de doenças, como a febre hemorrágica causada pelo vírus Ebola e outras doenças emergentes ou reemergentes, façam renascer o interesse pelos espaços naturais, inalterados ou muito pouco modificados (Burnet \& White, 1972; Croll \& Cross, 1983; Mayer, 1984; Pracontal, 1995; Robertson et al., 1996; Wills, 1996).

A rápida transformação verificada no terceiro mundo após a II Guerra Mundial, principalmente nas colônias em emancipação, foi reconhecida pelos epidemiologistas, que buscaram modelos teóricos para lidar com estas transformações. Foi um momento de surgimento, ou ressurgimento, da geografia médica, disciplina científica que data do século passado, mas que nunca se estabeleceu firmemente como distinta da epidemiologia. Vários autores, principalmente europeus e norte-americanos, recorreram à geografia para compreender o novo contexto epidemiológico internacional. Talvez o melhor exemplo seja o dos trabalhos de May, autor franco-americano, que buscou uma abordagem geográfica, mas de cunho antropológico, para compreender as alterações (May, 1958, 1977, 1978).

Exemplos mais recentes desta linha são inúmeros (Wildling et al., 1995) e partem da premissa que o meio natural já foi alterado pela ação humana, seja em período recente, ou mesmo pré-histórico. Ao mesmo tempo, emprestam um papel relevante às condições naturais, particularmente às climáticas.

Uma vez que o meio já não era 'natural', caberia recorrer a alguma forma de compreensão do comportamento humano. Executa-se aqui um sal to teórico da ecologia para a sociologia ou para a geografia humana (Amat-Roze, 1993). Este salto é feito inclusive por autores de influência marxista, como da escola mexicana de Laurell (Breihl, 1991). Todas estas análises relegam para um segundo plano a análise das transformações sofridas pelo espaço, mesmo quando existem transformações óbvias, como as hidrelétricas construídas na África que alteraram a epidemiologia de diversas doenças no terceiro mundo, como o clássico caso da in- fluência da construção da represa de Assuam e a disseminação da esquistossomose no Egito (Gordon-Smith, 1975; Hughes \& Hunter, 1970).

Quando se discutem doenças determinadas e geradas pela sociedade, como muitas das doenças do trabalho ou determinadas doenças mentais, é compreensível que se rel egue para um plano secundário a análise do espaço. Mas quando se busca a compreensão da epidemiologia de doenças muito ligadas ao meio, como a maioria das doenças infecciosas, particularmente as transmitidas por vetor, ou alguns cânceres determinados por exposição a substâncias existentes no meio, o espaço deve necessariamente entrar como categoria de análise, se não se quiser ofuscar processos importantes.

\section{A geografia marxista}

e o seu conceito de espaço

A corrente marxista na geografia (Santos, 1978a e b, 1979, 1988; Moraes \& Costa, 1984), notadamente dos geógrafos franceses como Pierre George (1972) e Olivier Dollfus (1972), trabaIhou a categoria espaço de uma maneira muito interessante para os epidemiologistas, mesmo os não marxistas.

A visão de Pierre George sobre a geografia pode ser facilmente transposta para a epidemiologia, no sentido em que ambas são disciplinas científicas cujo objeto é o centro de uma rede de relações por demais ampla e complexa para ser adequadamente compreendida através de uma visão metodológica estreita. Um pequeno trecho de suas reflexões sobre a geografia mostra bem esta visão:

“... a geografia tem de ser metodologicamente heterogênea. Alinha-se por um lado entreas ciências da terra ou da natureza, da mineralogi a e da petrografia, da geologia atéa biologia; por outro lado, a sociologia, a economia a psicologia social ... É esta a razão pela qual ela se encontra continuamente empenhada na busca de sua unidade. Esta uni dade não pode ser metodológi ca: a pesquisa geográfica recorresucessi vamente aos métodos de cada uma das ciências de que se vale para chegar ao conhecimento analítico dos dados incluídos nas combinações que constituem o objeto de seus estudos fragmentários ou globais" (George, 1972).

É fácil se perceber a analogia com a epidemiologia. George, como outros geógrafos, valese da análise do processo de organização do espaço como o esteio destas relações, dando coerência a um aparente caos. Tal análise é, portanto, um recurso metodológico, que pode 
perfeitamente ser transposto para a epidemiologia, onde a única mudança será o objeto, não mais a interação homem-meio, mas o processo saúde-doença.

Alguns conceitos são tão representativos e úteis em ciência, que mesmo que se derivem dos paradigmas que os originaram, ainda assim persistem. Alguns conceitos da psicologia analítica de Freud, como ego, ou o conceito marxista de classe social, sobrevivem em outros modelos ou teorias.

A leitura da teoria dos focos naturais de Pavlovsky, à luz dos conceitos geográficos de George e Dollfus, permite a elaboração de um conceito operacional de espaço extremamente rico para a análise epidemiológica. co é:

Segundo Dollfus (1972), o espaço geográfi-

“... um espaço mutável e diferenciado cuja aparência visível éa pai sagem. É um espaço recortado, subdividido, mas sempreem função do ponto de vista segundo o qual o consi deramos. Espaço fracionado, cujos el ementos se apresentam desi gual mente solidári os uns aos outros ... Por conseguinte, surge o espaço geográfico como o esteio de um sistema de relações, algumas determinadas a partir do mei o físico (arquitetura dos volumes rochosos, clima, vegetação), outras proveni entes das sociedades humanas responsáveis pela organização do espaço em função da densidade demográfica, da organização social e econômica, do nível das técnicas; numa palavra: de toda essa tessitura pejada de densidade histórica a que damos o nome de civilização".

Pavlovsky já trabal hava com a aparência visível do espaço, a paisagem. Sua teoria dos focos naturais deixa implícito que há um conjunto de relações no interior da paisagem responsáveis pela ocorrência da doença. Ocorre, como já notei acima, que Pavlovsky não levou sua teoria adiante. Seu conceito de mudança no espaço, levando à mudança na estrutura epidemiológica, ao ser transportado para o conceito de espaço geográfico de Dollfus, permite colocar a análise do espaço e de suas transformações como o ponto central da análise. O conceito de espaço geográfico incorpora os determinantes naturais e sociais numa visão de totalidade, que muitas vezes falta à análise epidemiológica. O espaço pode ser didaticamente dividido em três grandes categorias:

- o espaço natural, intocado: de pouco interesse para a epidemiologia, uma vez que não é utilizado pelas sociedades humanas. Pavlovsky, Audy e outros estudiosos da teoria dos focos naturais valorizavam o espaço natural por estarem interessados nas zoonoses;
- o espaço percorrido: alterado apenas ligeiramente pela ação humana, que não o explora; - o espaço organizado: alterado profundamente pela ação humana.

O determinante maior do processo de organização do espaço é a necessidade econômica, que vai reorganizar o espaço conforme as necessidades das atividades que devem se desenrolar, seja a agricultura, a exploração mineral, o transporte de mercadorias, a produção de energia, a fabricação de produtos ou a construção de cidades. Seja qual for esta atividade, determinará sobre o espaço um grau maior ou menor de organização. A sucessão histórica das necessi dades levará à superposição da organização espacial, tal como se percebe claramente na paisagem européia, onde se encontram vestígios da organização romana, do feudalismo, da revolução industrial e da época contemporânea (Hoskins, 1981). O mesmo se verifica com o espaço urbano, que é organizado e reorganizado sucessivamente. Os trabaIhos de Santos (1978a e b, 1979, 1988), geógrafo brasileiro, mostram bem o processo de organização do espaço urbano no terceiro mundo, conforme as necessidades da economia.

\section{A organização do espaço geográfico e a epidemiologia}

A análise do espaço geográfico em epidemiologia é particularmente interessante no momento atual, em que existe uma percepção maior da importância do meio ambiente sobre a existência da humanidade. A análise epidemiológica centrada sobre o espaço não deve ser entendida como uma visão ecologista do processo saúde-doença. A interpretação da epidemiologia como ecologia é parcial, não permitindo uma visão abrangente. Vários autores buscaram a vertente ecológica da epidemiologia (May, 1958; Barreto, 1967; Burnet \& White, 1972; Croll $\&$ Cross, 1983); entre estes, podemos destacar Fox (Fox et al., 1971), um autor norte-americano que entende a epidemiologia como a ecologia da doença. A ecologia tradicional é de cunho fortemente biológico, apenas recentemente incorporando outros sistemas de relações.

O presente interesse na ecologia exerce forte pressão para se entender a epidemiologia como ecologia. Na minha opinião, são disciplinas científicas distintas, ainda que com áreas comuns. Particularmente problemática é a conceituação de equilíbrio que permeia muitas análises ecológicas. A epidemiologia é essencialmente a disciplina que busca entender o desequilíbrio. 
O conceito de espaço geográfico, ainda que originário da geografia marxista, não necessariamente deve prender-se à interpretação marxista. A grande alteração teórica que a interpretação marxista introduziu com o conceito de espaço geográfico foi a passagem do centro de análise, tradicional mente no ser humano e na sociedade, para a interação sociedadenatureza. Uma mudança aparentemente sutil, mas que traz importantes contribuições teóricas. A epidemiologia se preocupa com o processo de ocorrência e distribuição das doenças nas coletividades, portanto o eixo de análise é a coletividade e seu comportamento. Se desviarmos ligeiramente nosso olhar e passarmos a analisar não mais a coletividade em si, mas o processo de interação desta com a natureza ea maneira como o meio é transformado, organizado para sustentar a atividade econômica, ganharemos uma perspectiva histórica da doença, fundamental para a compreensão do momento atual. A análise do processo de organização do espaço, por ser este um processo contínuo, permite uma visão dinâmica do processo saúde-doença. A análise do processo de organização do espaço é um recurso teórico em epidemiologia, não é de maneira al guma uma panacéia metodológica e tampouco substitui outras abordagens. Sua aplicação é mais produtiva na investigação das doenças infecciosas, o que não significa que não tenha outras aplicações, apenas tem sido mais utilizada neste campo (Silva, 1985, 1986, 1992).

Os escritos de Marx são inevitavelmente atraentes para os que se preocupam com o processo de organização do espaço e sua influência na ocorrência e distribuição das doenças. Um bom exemplo é um trecho da crítica de Marxe Engels a Ludwig Fuerbach, no qual, se substituirmos o exemplo da cerejeira por uma doença, como a dengue, a sua relação com a epidemiologia é evidente:

“Ele não vê que o mundo sensível que o rodeia não é um objeto dado di retamente de toda a eternidade e sem cessar semel hante a si próprio, maso produto da indústria e do estado da sociedade, e isto no sentido em que éo produto histórico, o resultado da atividade deuma série de gerações, em que cada uma se desembaraçava da precedente, aperfeiçoava a sua indústria e o seu comércio emodificava o seu regime social em função de transformações das necessi dades. Os objetos da mais si mples 'certeza sensível ' só são dados a Fuerbach pelo desenvolvimento social, a indústria e as trocas comerciais. Sabe-se que a cerejeira, como quase todas as árvores de frutos, foi transplantada para as nossas latitudes pelo comércio, há poucos séculos, e não foi, portanto, senão graças a esta ação de uma de terminada sociedade numa determinada época, que ela foi dada à 'certeza sensível' de Fuerbach" (grifo do autor) (Marx \& Engels, 1975).

A análise do processo de organização do espaço geográfico tem como ponto de partida o ponto de vista segundo o qual o consideramos. Este deve ser o centro do sistema de relações que será desvendado ao longo das investigações. O processo de análise é a reconstrução do sistema de relações que gira em torno do nosso objeto de estudo. O primeiro passo é a paisagem, a porção visível e aparente do espaço. Em epidemiologia, o ponto de vista que nos interessa será a doença, ou conjunto de doenças a ser investigado (Silva, 1985, 1986, 1992).

Muitas doenças, e em especial as zoonoses, têm habitats naturais em ecossistemas bem definidos nos quais patógenos, vetores e hospedeiros naturais formam associações, ou biocenoses, em que o patógeno circula. A paisagem é, assim, um fator epidemiológico, pois suas características são as do ecossistema local. A ocupação pelo homem de tais focos naturais leva à ocorrência de casos de doença no local. A doença passa a ter como que uma personalidade própria e se incorpora no contexto ecológico, sendo vista como parte integrante do ecossistema. Aqui se percebe a limitação da doutrina de Pavlovsky em explicar o comportamento de uma doença quando esta transcende o seu meio natural e se incorpora a uma sociedade humana, com um comportamento cuja explicação foge à ecologia somente.

Segundo Rougerie:

“Uma paisagem constitui um todo, percebido através de vários sentidos, e cujas relações causais, se desejarmos compreen dê-lo, deverão ser deslindadas uma por uma ..." (Rougerie, 1971).

A identificação destas relações causais ou fundamentais é a chave do processo de investigação. Uma vez feita esta identificação, passase para a reconstrução do processo de organização do espaço que resultou no sistema de relações identificado. Este espaço, cujo centro é o nosso objeto de estudo, pode ser denominado espaço nosológico. O recorte da totalidade feito do ponto de vista do epidemiologista colocará a doença em primeiro plano e buscará o sistema de relações que permite a ocorrência desta doença, não na interação humana como ponto de partida, mas na interação sociedadenatureza e nos modelos de interação humana dela decorrentes.

Dentro da perspectiva oferecida pela geografia, inverte-se o processo usual de análise em epidemiologia: ao invés de partir da doen- 
ça e analisar como esta se insere no contexto, parte-se da totalidade, analisando como esta criou as condições de ocorrência da doença. Com esta preocupação de analisar o problema com uma visão totalizadora, tomamos da Geografia o conceito de espaço geográfico, o que aliás já havia sido feito por Pavlovsky na sua epidemiologia paisagística.

No caso da doença de Chagas, uma doença estudada por meio da análise do processo de organização do espaço (Silva, 1985, 1986, 1992), a interação sociedade-natureza se manifesta pela maneira como a sociedade, em diferentes momentos históricos, realizou a produção agrária. O modelo econômico em si é por demais generalizante, uma vez que não fornece detalhes acerca do processo de organização do espaço.

O espaço dos geógrafos, ou pelo menos de determinados geógrafos, é mais denso e consistente, portanto mais rico que o espaço tradicional da epidemiologia. Este último é como o espaço da cólera em Londres, descrito por Snow (Snow, 1990), um espaço que tem como característica básica a origem da água utilizada. Este é um exemplo de espaço simplificado, que permite a compreensão de sistemas relativamente simples e delimitados.

\section{A esquistossomose mansônica como exemplo}

O espaço organizado e reorganizado não é inteiramente renovado, muitas vezes existem transformações ambientais profundas, que se refletem na existência de um contexto epidemiológico persistente, não obstante modificações subseqüentes profundas. Exemplo disto é a atual distribuição mundial da esquistossomose.

A esquistossomose, em diferentes momentos da antigüidade, estabeleceu-se nas regiões onde surgiram as chamadas civilizações de regadio, sociedades que dominaram a tecnologia da irrigação e conseguiram um excedente agrícola que levou à edificação de cidades e à expansão de impérios.

Estas civilizações, que surgiram em diferentes locais e em diferentes momentos da história, aprenderam a aproveitar a água de grandes rios, geralmente em planícies inundáveis, para a irrigação perene de suas terras; isto, juntamente com o aluvião resultante de enchentes periódicas, permitia o uso do mesmo solo para agricultura por anos a fio (Ribeiro, 1968).

A distribuição destas civilizações coincide com a distribuição da esquistossomose. Este fato não é de se estranhar, pois a presença de sistemas de irrigação é considerada como fator importante na ocorrência da esquistossomose.

Um sistema de canais de irrigação oferece não só uma infinidade de habitats para os caramujos, como, por força da atividade desenvolvida, coloca o homem em contato constante com a água, uma vez que canais de irrigação demandam manutenção permanente.

Ao longo da história, várias destas civilizações de regadio surgiram - desde o Império Acádio, na Mesopotâmia, por volta de 2350 a.C., até a dinastia Togugawa, no Japão, já no século 17 d.C. - e todas se estruturaram às margens de grandes rios, como o Tigre e o Eufrates na Mesopotâmia, o Nilo no Egito, o Mekong no Camboja e o Yang-tze-kiang na China.

Ainda que não existam grandes rios no Japão, há referências à irrigação já nas lendas sobre os primeiros governantes japoneses. A interação entre o Japão e a China data pelo menos do início da era cristã, sendo possivelmente mais antiga.

Estes impérios tiveram seu período de glória, e de decadência, mas a marca deixada no meio ambiente foi importante, tanto que nestas regiões, de uma forma ou de outra, continua sendo praticada uma agricultura de irrigação.

Em cada uma destas regiões, num dado período histórico, a esquistossomose ou foi introduzida e encontrou condições ideais para a sua disseminação, ou efetivamente surgiu, a partir de zoonoses.

Não há necessidade de lembrar que estas civilizações já desapareceram há muito, no entanto, as transformações ambientais que determinaram, assim como a herança da transmissão da esquistossomose, persistem.

A análise do espaço geográfico não tem interesse apenas quanto à história de uma dada doença, ainda que esta seja fundamental para a compreensão do momento presente.

O espaço é o cenário onde se desenvolvem as interações entre os diferentes segmentos das sociedades humanas e entre estas e a natureza. As doenças surgem ou, pelo menos, são modificadas por estas interações. Compreender o processo de organização do espaço pelas sociedades humanas em diferentes momentos e lugares é uma forma particular de entender as estas sociedades. Para a epidemiologia, a compreensão do processo de organização do espaço permite entender o papel do natural na gênese e distribuição das doenças. 
O problema atual da urbanização no terceiro mundo

No momento presente, e no futuro próximo, o espaço urbano, forma elaborada de organização do espaço, é e será o cenário dos principais desafios no caminho para o controle das doenças, infecciosas ou não (World Health Statistics Quarterly, 1991). A população mundial, estimada em 4.851 milhões em 1985, deverá atingir 6,2 milhões no ano 2000 e 8.504 milhões em 2025, um crescimento de 29\% entre 1985 e 2000 e de $36 \%$ entre 2000 e 2025 . A população urbana, por outro lado, estimada em $2.048 \mathrm{mi}$ Ihões em 1985, deve atingir 3,1 milhões no ano 2000 e 5.493 milhões em 2025, um crescimento de $56 \%$ e $72 \%$, respectivamente.

Este crescimento deverá ser verificado de maneira mais acentuada nos países em desenvolvimento. Em 1970, havia 62 aglomerados urbanos com mais de dois milhões de habitantes; em 1985 eram 99, sendo cinqüenta destes em países em desenvolvimento. As características destes aglomerados urbanos são diferentes ao longo de todo o mundo subdesenvolvido, comum a eles talvez só a enorme quantidade de problemas. Este crescimento acelerado se faz através de processos de organização do espaço os mais variados, impossíveis de ser generalizados e instáveis em relação ao tempo. Isto exige uma metodologia de análise que contemple estas variações.

Ainda que os primeiros investigadores dentro da linha da teoria dos focos naturais de Pavlovsky buscassem identificar padrões paisagísticos que permitissem antever as doenças existentes, a aceleração da intervenção humana no espaço natural se fez de maneira tão rápida, criando novos e mutáveis padrões de organização espacial, que o tratamento dado por Pavlovsky ao espaço se tornou rapidamente obsoleto, uma vez que o espaço natural praticamente não existe mais. Além da necessidade de se contar com uma metodologia adequada para dar conta das rápidas transformações verificadas no espaço urbano e seus reflexos sobre a ocorrência e distribuição das doenças, esta mesma metodologia deve subsidiar o planejamento dos sistemas de atenção à saúde para estes mesmos centros urbanos.

Se analisarmos boa parte das publicações acerca do impacto da urbanização sobre o processo saúde-doença, veremos que não existe uma preocupação de se analisarem as particularidades de cada centro urbano, mas sim uma tentativa de generalização, incorrendo num simplismo semelhante ao existente em épocas recentes acerca das endemias rurais, quando a mai oria dos autores afirmava que estas - doença de Chagas, ancilostomíase, esquistossomose, bócio endêmico, malária e outras - ocorriam devido às:

“... vergonhosas condições de existência que leva uma grande parte da população rural dos países da América Latina" (Martins, 1968).

Não se trata aqui de negar serem lamentáveis as condições de vida na zona rural da América Latina, mas sim de apontar para o caráter excessivamente simplificante destas afirmações, que acabam encobrindo as particularidades de cada região e período histórico. A análise do espaço enquanto categoria epidemiológica surge como uma necessidade, tanto no passado, quando a grande problemática de saúde, particularmente no que diz respeito às doenças infecciosas, estava na zona rural, como no momento presente, com a urbanização acelerada que se verifica no terceiro mundo (Heinmann et al., 1992).

Talvez a mais representativa destas seja o último número de 1991 do World Health Statistics Quarterly (World Health Statistics Quarterly, 1991), totalmente dedicado aos problemas determinados pela rápida urbanização do terceiro mundo e que expressa, naturalmente, a posição da Organização Mundial da Saúde. Não é necessária uma análise muito profunda para se perceber que o paradigma explicativo utilizado é o do crescimento populacional descontrolado.

Perspectivas do uso do conceito de organização do espaço em epidemiologia das doenças infecciosas

A utilização da categoria espaço em epidemiologia é um recurso de investigação, por meio do qual se muda o eixo de investigação. Não é, portanto, uma metodologia pronta e acabada. O espaço pode receber diferentes definições e interpretações. A utilização cada vez maior de model os científicos em que o caráter imprevisível dos fenômenos é valorizado, como o caos determinista ou a teoria das catástrofes (Arnold, 1989; Ekeland, 1987; Science Illustrée, 1996), tem a sua contrapartida na geometria. A geometria dos fractais e a teoria do caos têm sido cada vez mais utilizadas nas ciências naturais, na medicina inclusive, sendo a base de estudos sobre o contágio (Hénon, 1989). Não é minha intenção analisar estas evoluções, quero somente lembrar que a utilização da categoria espaço como recurso de investigação em epidemiologia não se prende a paradigmas ou metodologias, podendo sofrer modificações conforme a evolução dos modelos utilizados, 
tendo, portanto, um grande potencial de evolução, cujo aproveitamento já vem sendo feito (Sabroza et al., 1995). Cabe destacar a utilização de satélites de sensoreamento remoto para a criação de bases de dados para estudos epidemiológicos, representando talvez um reencontro da epidemiologia com a geografia (Beyers et al., 1996).

\section{Referências}

AMAT-ROZE, J. M., 1993. Les inegalites geographiques de l'infection a VIH et du SIDA en Afrique sud-saharienne. Social Science and Medicine, 36:1.247-1.256

ARNOLD, V. L., 1989. Singularidade dos contornos de estabilidade e o princípio da fragilidade do que é bom. In: Teoria da Catástrofe (V. L. Arnold, org.), pp. 55-57, Campinas: Editora da Unicamp.

AUDY, J. R., 1958. The localization of disease with special reference to the zoonoses. Transactions of the Royal Society of Tropical Medicine and Hygiene, 52:308-328.

BARRETO, M. P., 1967. Aspectos ecológicos da epidemiologia das doenças transmissíveis, com especial referência às zoonoses. Revista Brasileira de Malariologia e Doenças Tropicais, 19:633-654.

BEYERS, N.; GIE, R. P.; ZIETSM AN, H. L.; KUNNEKE, M.; HAUMAN, J.; TATLEY, M. \& DONALD, P. R., 1996. The use of a geographical information system (GIS) to evaluate the distribution of tuberculosis in a high-incidence community. South African Medical Journal, 86:40-44.

BREIHL, J., 1991. Epidemiologia. Economia, Política e Saúde. São Paulo: Editora da Unesp/Hucitec.

BUCK, C.; LLOPIS, A.; NÁJERA, E. \&TERRIS, M., 1988. El Desafio de la Epidemiologia. Problemas y Lecturas Sel ecionadas. Washington: Organizacion Panamericana de la Salud. Publ. Cient. n. 505.

BURGOS, J. J.; CURTO DE CASAS, S. I.; CARCAVALLO, R. U. \& GALINDEZ, G. I., 1994. Global climate change in the distribution of some pathogenic complexes. Entomologia y Vectores, 1:69-82.

BURNET, M. \& WHITE, D. O., 1972. The ecological point of view. In: Natural History of Infectious Disease (M. Burnet $\&$ D. O. White, eds.), 4th ed., pp. 1-21, Cambridge: Cambridge University Press.

COIMBRA JR., C. E. A.; SANTOS, R. V. \& SMANIO-NETO, L., 1984. Potencial endêmico da esquistossomose para o Estado de Rondônia, Brasil. Revista deSaúdePública, 18:510-515.
CROLL, N. A. \& CROSS, J. H., 1983. Human Ecology and Infectious Diseases. New York: Academic Press.

DOLLFUS, O., 1972. O Espaço Geográfico. São Paulo: Difusão Européia do Livro.

EKELAND, I., 1987. A volta da geometria. In: O Cálculo e o Imprevisto (I. Ekeland, org.), pp. 81-114, São Paulo: Martins Fontes.

FORATTINI, O. P., 1976. Epidemiologia Geral. São Paulo: Edgard Blücher/Edusp.

FOX, J. P.; HALL, C. E. \& ELVEBACK, L. R., 1971. Epidemiology - Man and Disease. New York: MacMilIan Co.

GEORGE, P., 1972. Os Métodos da Geografia. São Paulo: Difusão Européia do Livro.

GILL, C. A., 1920a. The relationship between malaria and rainfall. Indian Journal of Medical Research, 37:618-632.

GILL, C. A., 1920b. The role of meteorology and malaria. Indian Journal of Medical Research, 8: 633-693.

GORDON-SMITH, C. E., 1975. Changing patterns of disease in the tropics. In: Man - Made Lakes and Human Health (N. F. Stanley \& M. P. Alpers, eds.), pp. 345-362, London: Academic Press.

HEINMANN, L. S.; CARVALHEIRO, J. R.; DONATO, A.; IBANHES, L. C.; LOBO, E. F. \& PESSOTO, U. C., 1992. Epidemiologia, condições de vida e território. In: O Município ea Saúde (L. S. Heinmann, J. R. Carvalheiro, A. Donato, L. C. Ibanhes, E. F. Lobo \& U. C. Pessoto, orgs.), pp. 26-32, São Paulo: Hucitec.

HÉNON, M., 1989. La diffusion chaotique. La Récherche, 20:490-498.

HEUNIS, J. C.; OLIVIER, J. \& BOURNE, D. E., 1995. Short-term relationships between winter temperatures and cardiac disease mortality in Cape Town. South African Medical Journal, 85:1.0161.019 .

HOSKINS, W. G., 1981. The Making of the English Landscape. Harmondsworth: Penguin Books. 
HUGHES, C. C. \& HUNTER, J. M., 1970. Disease and "development" in Africa. Social Sciences and Medicine, 3:443-493.

LOVEJOY, T. E., 1993. Global change and epidemiology: nasty synergies. In: Emerging Viruses (S. E. Morse, ed.), pp. 281-268, New York/ Oxford: Oxford University Press.

MARTINS, A. V., 1968. Epidemiologia. In: Doença de Chagas (J. R. Cançado, org.), pp. 225-260, Belo Horizonte: Imprensa Oficial.

MARX, K. \& ENGELS, F., 1975. A ideologia alemã. In: Ludwig Fuerbach e o Fim da Filosofia Clássica Alemã (K. Marx \& F. Engels, orgs.), pp. 25-30, Lisboa: Editorial Estampa.

MAY, J. M., 1958. The Ecology of Human Disease. New York: M D Publications.

MAY, J. M., 1977. Medical geography: its methods and objectives. Social Science and Medicine, 11:715730.

MAY, J. M., 1978. History, definitions and problems of medical geography: a general review. Social Science and Medicine, 12D:211-219.

MAYER, J. D., 1984. Medical geography. An emerging discipline. Journal of the American Medical Association, 251:2680-2685

MORAES, A. C. R. \& COSTA, W. M., 1984. Uma teoria marxista da geografia. In: Geografia Crítica. A Valorização do Espaço (A. C. R. Moraes \& W. M. Costa, orgs.), 2a ed., pp. 35-46, São Paulo: Hucitec.

MORSE, S. E., 1995. Factors in the emergence of infectious diseases. Emerging Infectious Diseases, 1(1):http:// www.cdc.gov/ncidod/EID/vollnol/ morse.htm

SCIENCE ILLUSTRÉE, 1996. Nous vivons au bord du chaos. Sciencelllustrée, 12:50-55

PAVLOVSKY, E. N., (s/data.)a. Natural Nidality of Transmissible Diseases. Moscou: Peace Publishers.

PAVLOVSKY, E. N., (s/ data.)b. The current status of the theory of natural focality of human diseases. In: Human Diseases with Natural Foci (E. N. Pavlovsky, ed.), pp. 9-44, Moscow: Foreign Languages Publishing House.

PEIXOTO, A., 1975. Clima e Saúde. Introdução Biogeográfica à Civilização Brasileira. São Paulo: Companhia Editora Nacional.

PESSOA, S. B., 1978. Ensaios Médico-Sociais. 2a ed., São Paulo: Cebes/ Hucitec.

PRACONTAL, M., 1995. Les règles de l'ecologie virale. Science et Vie, Decembre:80-84.

RIBEIRO, D., 1968. O Processo Civilizatório. Rio de Janeiro: Civilização Brasileira.

ROBERTSON, S. E.; HULL, B. P.; TOMORI, O.; BELE, O.; LEDUC, J. W. \& ESTEVES, K., 1996. Yellow fever. A decade of reemergence. Journal of the American Medical Association, 276:1.157-1.162.
ROU GERIE, G., 1971. Geografia das Paisagens. São Paulo: Difusão Européia do Livro.

SABROZA, P. C.; KAWA, H. \& CAM POS, W. S. Q., 1995. Doenças transmissíveis, ainda um desafio. In: Os Muitos Brasis. Saúdee População na Década de 80 (M. C. S. Minayo, org.), pp.177-244, São Paulo/Rio de Janeiro: Hucitec/ Abrasco.

SANTOS, M., 1978a. Por uma Geografia Nova. São Paulo: Edusp/Hucitec.

SANTOS, M., 1978b. Pobreza Urbana. São Paulo: Hucitec.

SANTOS, M., 1979. O Espaço Dividido. Os Dois Circuitos da Economia Urbana nos Países Subdesenvolvidos. Rio de Janeiro: Francisco Alves.

SANTOS, M., 1988. O Espaço em Questão. São Paulo: Marco Zero/AGB.

SAVOIA, A.; ZATTERALE, A.; DEL PRINCIPE, D. \& JOENJE, H., 1996. Fanconi anaemia in Italy: high prevalence of complementation group $A$ in two geographic clusters. Human Genetics, 97:599-603.

SILVA, L. J., 1985. Crescimento urbano e doença. A esquistossomose no Município de São Paulo (Brasil). Revista deSaúdePública, 19:1-7.

SILVA, L. J., 1986. Desbravamento, agricultura e doença: a doença de Chagas no Estado de São Paulo. Cadernos deSaúde Pública, 2:124-140.

SILVA, L. J., 1992. Organização do espaço e doença. In: Textos de Apoio. Epidemi ol ogia I (J. R. CarvaIheiro, ed.), vol. 1, 2a ed., pp. 59-85, Rio de Janeiro: Escola Nacional de Saúde Pública.

SINHA, T. \& BENEDICT, R., 1996. Relationship between latitude and melanoma incidence: international evidence. Cancer Letters, 99:225-231.

SNOW, J., 1990. Sobre a Maneira de Transmissão do Cólera. São Paulo/Rio de Janeiro: Hucitec/ AbrasCo.

VERHASSELT, Y., 1981. The contribution and future development of spatial epidemiology. Social Science and Medicine, 15A:333-335.

WILDING, E.; WINKLER, S.; KREMSER, P. G.; BRANDTS, C.; JENNE, L. \& WERNSDORFER, W. H., 1995. Malaria epidemiology in the province of Moyen Ogov, Gabon. Annals of Tropical Medicine and Parasitology, 46:77-82.

WILLS, C., 1996. Yellow Fever, Black Goddess: The Coevolution of People and Plagues. Reading: Addison-Wesley.

WORLD HEALTH STATISTICS QUARTERLY, 1991. Urbanization and health in developing countries: a challange for health for all. World Health Statistics Quarterly, 44:186-244. 